\title{
BUTH SAVONG Y LA NUEVA PROLIFERACIÓN DE RELIQUIAS EN CAMBOYA
}

\author{
JOHN MARSTON \\ El Colegio de México
}

Los especialistas en budismo camboyano contemporáneo y los intelectuales camboyanos, tanto como los observadores extranjeros, si no es que más, suelen reprobar el estado actual del budismo en el país, y refieren lo que consideran una falta de moralidad, de educación budista y de disciplina monástica. La posición refleja la desorientación y la pérdida cultural del país, ambas muy reales, en una época de profundo cambio sociopolítico; sin embargo, he argumentado que es una visión demasiado simplista de las contradicciones y las ironías de la práctica religiosa, así como quizás de la lógica del cambio social. El principal líder-maestro religioso laico del país, Buth Savong, y el movimiento que lo rodea, se yerguen notoriamente como un locus de inercia social para los budistas camboyanos que tratan de abordar problemas como los que plantean estos críticos. Sin importar cuáles sean las preguntas que se hagan sobre él y su movimiento, hay que reconocerlo como alguien alrededor de quien se depositan energías religiosas y se generan síntesis culturales, la cristalización de una visión de lo que puede ser el budismo camboyano.

Existen diversas maneras de aproximarse al fenómeno de Buth Savong: el análisis de sus enseñanzas reales, que se concentran principalmente en la explicación del Abhidhamma; su enfoque sobre la implementación de la disciplina monástica; su uso de la radio como un medio, y la forma en que las redes nacionales e internacionales han generado fama y apoyo finan-

Este artículo fue recibido por la dirección de la revista el 5 de marzo de 2013 y aceptado para su publicación el 14 de mayo de 2013. 
ciero. En el presente artículo discuto la importancia de la manera en que se organizan las estructuras físicas en los wat (complejos de templos) y en los asram (complejos organizados menos formalmente) asociados con él, y en particular la importancia de las reliquias y las stupas de las reliquias que se encuentran ahí; un tipo de reforma que, sostengo, implica nuevas formas de configurar el espacio sagrado en los sitios religiosos camboyanos, o incluso un nuevo mapa de lo sagrado, según se proyecta en la visión de la nación camboyana.

Este estudio trata específicamente sobre los sucesos que se han dado en Camboya, pero puede decirse que se relaciona con un interés académico más general: la función de las reliquias budistas según se ha desarrollado en los estudios budistas y en otras disciplinas a lo largo de los últimos 20 años, parte de lo que Trainor ha llamado la "rematerialización" de la tradición. ${ }^{1}$ Robert Sharf incluso ha argumentado en términos históricos:

Más que visualizar la expansión del budismo a través de Asia como la propagación de un credo sagrado o una fe, el movimiento del budismo puede entenderse mejor en términos de la difusión de objetos sagrados, sobre todo los iconos y las reliquias, junto con el conocimiento técnico esotérico requerido para manipularlos. ${ }^{2}$

Me baso en una tradición académica que considera que las reliquias o las stupas que las resguardan asumen una importancia cosmológica, y que las configuraciones políticas inevitablemente comparten aspectos de las cosmologías que las reliquias configuran (y buscan en ellas legitimación). Académicos como Wolters ${ }^{3}$ y Tambiah $^{4}$ han explorado la prominencia de tales configuraciones en la entidad política premoderna del sureste

${ }^{1}$ Kevin Trainor, Relics, Ritual, and Representation in Buddhism: Rematerializing the Sri Lankan Theravada Tradition, Cambridge-Nueva York, Cambridge University Press, 1997; y Kevin Trainor, "Introduction beyond Superstition", en D. Germano y K. Trainor (eds.), Embodying the Dharma: Buddhist Relic Veneration in Asia, Albany, State University of New York Press, 2004.

${ }^{2}$ Robert H. Sharf, "On the Allure of Buddhist Relics”, en Germano y Trainor (eds.), Embodying the Dharma, op. cit., p. 165.

${ }^{3}$ O. W. Wolters, History, Culture, and Region in Southeast Asian Perspectives, ed. rev., Ithaca, Cornell University Southeast Asia Program Publications, 1999.

${ }^{4}$ Stanley Tambiah, World Conqueror and World Renouncer: A Study of Buddhism and Polity in Thailand against a Historical Background, Cambridge, Cambridge University Press, 1976. 
asiático; esto no constituye una relación estática, sino una que se instituye de manera performativa con grados de efectividad en momentos históricos.

Apunto incidentalmente la observación de Strong de que, incluso en la época de Asoka (304-232 a.n.e.; el emperador más celebrado de la dinastía Maurya de India, y posteriormente un modelo para la forma en que definió el Estado budista), la cosmología de las stupas tenía tendencias tanto centrífugas como centrípetas, una tensión que quizás podría decirse que figura también en la nueva dinámica de la veneración de reliquias en la Camboya contemporánea. ${ }^{5}$

El interés de Buth Savong en instalar reliquias en Camboya coincidió con el periodo en el que, con apoyo del Estado, se estaba construyendo una descomunal stupa en la montaña de Reachtrop, cerca de Oudong, para alojar reliquias de importancia histórica que se encontraban en una stupa frente a la estación de ferrocarril de Phnom Penh, proyecto que capturó la imaginación milenaria de algunos camboyanos. ${ }^{6}$ Su campaña coincide con un mayor movimiento internacional de reliquias: un nuevo transnacionalismo de reliquias. ${ }^{7}$

Buth Savong nació en el distrito de Sangker, cerca de la ciudad provincial de Battambang, en el occidente de Camboya, en 1960. Ahí acudió a la escuela primaria y se encontraba en secundaria en la ciudad provincial cuando se le evacuó al campo tras la caída del país en manos del Khmer Rojo. ${ }^{8} \mathrm{Su}$ madre murió durante esos años. Se ordenó como novicio en el periodo posterior a Pol Pot. Aunque logró vivir como monje en un templo local durante cuatro años, finalmente cedió ante las restricciones del gobierno contra los monjes jóvenes y colgó los hábitos; no obstante, en muchos sentidos continuó viviendo

${ }^{5}$ John S. Strong, Relics of the Buddha, Princeton, Princeton University Press, 2004, pp. 142-144.

${ }^{6}$ Una stupa es una estructura religiosa budista diseñada para alojar reliquias del Buda o de otras figuras religiosas importantes (en un sentido más informal, el término puede referirse a estructuras más pequeñas cuya función es alojar los restos de budistas laicos). En la tradición theravada, las stupas suelen tener una estructura cónica.

${ }^{7}$ Tal afirmación se basa en las pruebas que se presentan en este artículo, así como en reportes sobre nuevas reliquias en el sur de Vietnam y en Estados Unidos, y en las giras internacionales de reliquias tibetanas que se han llevado a cabo recientemente.

${ }^{8}$ Buth Savong, Parents' Words of Wisdom, Phnom Penh, Dhamma4khmers. org, 2005. 
como un monje. Su desarrollo como estudiante de budismo y, según lo que se dice, su gran dominio del pali, la lengua en la que se encuentran las escrituras budistas, se derivan en parte de la instrucción dhamma (es decir, en este contexto, la enseñanza de las escrituras y las doctrinas budistas) que escuchaba en la radio tailandesa en los años ochenta (y quizás, de acuerdo con un académico estadounidense que realizaba entrevistas sobre él, del tiempo que pasó estudiando en wat tailandeses). ${ }^{9} \mathrm{De}$ manera un tanto similar a la figura de Anagarika Dharmapala, en Sri Lanka, ha dedicado su vida al estudio del dhamma, viste de blanco (no las túnicas color azafrán de los monjes), se afeita la cabeza y, según se dice, sigue las reglas disciplinarias monásticas de manera más estricta que la mayoría de los monjes. Un esbozo biográfico en una versión en lengua inglesa de uno de sus libros dice que empezó a enseñar el dhamma en Battambang, en 1980 (cuando era monje), y el Abhidhamma en Phnom Penh, en 1997.

En 1990 surgía ya como una figura carismática cuando, como recuerda un académico australiano, oficiales del Frente lo refirieron como una fuente de información sobre las creencias y las prácticas religiosas camboyanas. ${ }^{10}$ En ese momento, su plataforma consistía en casas individuales, ya sea en Battambang o en Phnom Penh, que visitaba frecuentemente y en las que solía conversar durante varias horas. Una buena parte de sus seguidores eran mujeres de diversas edades, no sólo las ancianas que comúnmente se apegan a las figuras de alto rango del monacato.

Buth Savong se perfiló como un maestro de dhamma, de renombre en la radio a mediados de la década de 1990, cuando, tras las elecciones patrocinadas por Naciones Unidas y el alejamiento del socialismo, los medios de comunicación se abrieron de manera importante. También puede resultar significativo que su ascenso a la prominencia ocurriera en el periodo posterior a 1989 , cuando el gobierno camboyano levantó las restricciones contra la ordenación de monjes jóvenes y los wat quedaron bajo la autoridad de un nuevo Ministerio de Religión, ya no en manos del Frente socialista. Aunque en

${ }^{9}$ Entrevista con Peter Gyallay-Pap, 15 de junio de 2011.

${ }^{10}$ Comunicación personal, Maurice Eisenbruch, junio de 2012. 
ese momento no decidió volver a ordenarse, atrajo el interés de algunos de los monjes más jóvenes que tenían una vocación budista genuina, y además adquirió una gran base de seguidores, principalmente de la generación de personas mayores de 30 años que recordaban el budismo camboyano tal como era antes del periodo del Khmer Rojo. Hasta 1997, Buth Savong vivió en la pequeña casa de madera de Battambang a la que aún regresa durante la temporada anual de lluvias. (La casa sigue siendo un símbolo de ascetismo, aunque ahora forma parte de un intrincado complejo budista con elaboradas estatuas, un salón techado para reuniones masivas, salones de clase y santuarios separados para alojar las escrituras budistas y una reliquia del Buda.) Las autoridades monásticas (quizás encabezadas principalmente por Samdech Oum Sum, uno de los monjes de más alto rango en ese momento) y el primer ministro Hun le otorgaron el título de akkeapoeundit (doctor) en la época en la que se mudó a Phnom Penh, a mediados de la década de 1990. Ahora habita una sencilla vivienda estilo casa-tienda china cerca del mercado de Tuol Tompong. Cuando está en Phnom Penh da pláticas cuatro días a la semana, dos de ellos en el wat Unnalom y dos en el wat Samphiw Meas, y otorga atención especial a la explicación de las escrituras budistas. Todas las charlas de Buth Savong atraen a un gran número de personas, con audiencias que se desbordan en los dos wat de Phnom Penh y públicos aún mayores cuando habla en otros lugares del país. De acuerdo con sus seguidores tenía (ya en 2006) unas 2000 cintas con sermones grabados, que se distribuyen gratuitamente o bien pueden comprarse. Las grabaciones de sus sermones aparecen con frecuencia en un sitio de Facebook que suele publicar sermones en lengua khmer. Asimismo, ha escrito varios libros de sermones inspiradores y poesía.

Si bien los libros y las pláticas de dhamma que he tenido la oportunidad de ver no hacen un llamamiento significativo a la reforma, se nota definitivamente que su insistencia en una mayor pureza representa un elemento de reforma en el budismo camboyano. Su peculiar función como un asceta que no es monje lo coloca en posición para criticar al monacato, en un momento en el que muchos se encuentran insatisfechos con él y consideran que carece de las cualidades que tenía antes 
del periodo de Pol Pot. Así, en cierto sentido es monje pero también está fuera del monacato y por encima de él. Se le ha asociado de cerca con el llamado a la reforma disciplinaria. Los wat vinculados con él adoptan un control disciplinario mucho más estricto que la norma camboyana. En Battambang, los wat asociados con él se denominan vinaya. Dado que los wat afiliados con él son mahanikay (la mayor de las dos órdenes monásticas que hay en Camboya), resulta significativo que algunas de las reformas en las que insiste, como que los monjes no usen calzado ni manejen dinero, ya son comunes en la práctica disciplinaria de la orden menor, thammayut; sin embargo, él no propugna recurrir a la thammayut, sino a sus propios conceptos de pureza disciplinaria.

Comenzamos a ver la complejidad de lo que representa cuando notamos las situaciones en las que los wat se han dividido, entre las facciones que desean seguir a Buth Savong y sus reformas y las que no. Hay muchas similitudes con las tensiones en Camboya, a lo largo del periodo colonial y la independencia hasta el periodo de Pol Pot, entre los elementos reformados (samey) y los tradicionales (boran) del budismo camboyano. Según lo que sé, esto sólo ocurrió en unas cuantas situaciones; por ejemplo, en el wat Sophi, en Battambang, uno de los primeros donde monjes importantes se interesaron en Buth Savong, y un caso que he podido observar en el distrito de Mchul Kiri, Kampong Chhnang, que quizás puede considerarse una parte natural de cualquier movimiento de reforma. Esto no se ha convertido en una perturbación significativa para el budismo camboyano, pero es interesante en la medida en que revela algunas de sus líneas de falla subyacentes. ${ }^{11}$

Al parecer, la discusión sobre Buth Savong incluye invariablemente algún comentario sobre su filiación política; aunque como maestro de dhamma no hace referencias políticas, se dice que tiene una cómoda posición en el seno del Partido Popular

${ }^{11}$ De acuerdo con Peter Gyallay-Pap, en la controversia en el wat Sophi, a la facción de Buth Savong se le conocía como el grupo vinaya y se le tenía por opuesto al grupo samey (moderno). En contraste, en Mchulkiri, se consideraba que el grupo que apoyaba a Buth Savong era contrario a aquellos que deseaban seguir las costumbres boran (tradicionales-antiguas). Véase John Marston, "La reconstrucción de budismo 'antiguo' de Camboya”, Estudios de Asia y África, vol. XxxvII (2), núm. 118, 2002, pp. 271-303. 
de Camboya (PPC), que se encuentra en el poder; algunos observadores afirman que predica la sumisión a la autoridad. Un alto funcionario del PPC fue fundamental en la recaudación de fondos que se realizó para sostener el ritmo constante de sus transmisiones radiales por todo el país. En una visita a uno de sus principales wat, en 2011, vi la imagen de los tres grandes líderes del PPC en el muro del dormitorio de un monje. No hay duda de que gran parte de los fondos que sostienen los proyectos de construcción de los wat y los asram afiliados con él, incluidas las stupas para reliquias que son el punto focal de este artículo, provienen de personas muy cercanas al PPC. (Y si su movimiento ha perdido apoyo popular, puede deberse a la percepción creciente de que él tiene vínculos muy cercanos con los poderosos o que incluso lo han cooptado.) Éste no es el primer caso en el que el aumento del institucionalismo implica una conexión creciente con los poderes reinantes; no obstante, yo argumentaría que su atractivo radica en una relación muy compleja con la autoridad. Por último, él representa a alguien que se vio forzado a abandonar el monacato, y gran parte de su atractivo se ha debido a la crítica honesta de un monacato que alguna vez lo excluyó. Si bien sus metas quizás no sean particularmente incongruentes con las de las autoridades estatales, definitivamente es un reformador desestabilizante que prospera en un entorno de agravio y avidez de una mayor pureza y orden.

Como ocurre con otros líderes religiosos, una parte importante de su atractivo sobre la cual es muy difícil escribir es el "aura" que emana. Cualquier evaluación de ese tipo es subjetiva, pero me aventuraré a decir que actualmente es el líder budista camboyano que, al menos en mi opinión, transmite más intensamente un sentido de espiritualidad, aunque esta clase de aseveraciones inevitablemente plantea preguntas sobre en qué consiste esa aura y cómo se ha creado. Muchos observadores concluyen que Buth Savong debe tener una intensa experiencia personal con la meditación, aunque en sus enseñanzas no la destaca, y es posible que su aura se deba a otros tipos de prácticas ascéticas. A riesgo de otorgar una mayor prioridad a mi propia percepción que a la de los camboyanos mismos, sugeriría contrastar a otro gran líder espiritual camboyano, Maha 
Ghosananda (que tuvo que ver particularmente con una serie de marchas por la paz en la década de 1990), ${ }^{12}$ cuya aura evocaría una gozosa apertura a nuevas posibilidades en la búsqueda de la paz, con Buth Savong, cuya aura, bastante más sepulcral, sugiere que su concentración de pureza espiritual brinda una manera de poner en perspectiva un gran sufrimiento.

Aunque creo que él nunca ha tenido grandes confrontaciones con las autoridades monásticas camboyanas, resulta significativo que haya tres puntos en los que parece haber divergido de la política monástica oficial. En primer lugar (aunque no cuento con documentación clara al respecto), algunos recuentos indican que ha insistido en emplear la versión tailandesa de las escrituras en pali en lugar de la versión khmer autorizada. En segundo lugar, las autoridades monásticas decidieron que los wat de Buth Savong no podían pedir a los monjes mahanikay que iban a residir en ellos que se ordenaran nuevamente, como a él le hubiera gustado, pues ello efectivamente crearía una nueva orden monástica en Camboya. En tercer lugar, el consejo monástico falló específicamente que sólo existe un sitio que alberga reliquias del Buda en Camboya, Sakyamunichedi en la montaña Reachtrop, que todas las demás reliquias deben colocarse en la stupa central en dicho sitio y que cualesquiera otras "reliquias" son inválidas. A pesar de este pronunciamiento, Buth Savong y el movimiento que lo rodea siguen llevando reliquias al país. Podría decirse que el proyecto estatal de instalar una reliquia en una stupa central en Oudong representaba la lógica de las tendencias centrípetas de una reliquia, mientras que la importancia misma que se otorga a la stupa de las reliquias, al hacerlo, condujo inevitablemente a las tendencias centrífugas representadas en la manera en que se han multiplicado las reliquias en el movimiento de Buth Savong.

Cuando uno comienza a visitar los wat y los asram vinculados con Buth Savong, empieza a notar ciertos patrones que los diferencian de la mayoría de los otros wat camboyanos. Después de visitar el wat Sokharam Tromoung en el distrito de Memot, Kampong Cham, y ver inscrita en mármol una lista

\footnotetext{
${ }^{12}$ Katherine Poethig, "Locating the Transnational in Cambodia's Dhammayātrā", en John Marston y Elizabeth Guthrie (eds.), History, Buddhism, and Nerw Religious Movements in Cambodia, Honolulú, University of Hawai'i Press, 2004, pp. 197-212.
} 
de los requisitos que el wat había cumplido, según la tradición de Asoka, para ser considerado sitio de adoración (Bochaniyathan), me di cuenta de lo explícito que esto era para la concepción de un lugar sagrado según se practica en los wat y los asram afiliados con Buth Savong. Los elementos que conformaban la lista de la inscripción eran:

1) Tripitaka.

2) Reliquia del Buda.

3) Stupa de reliquias.

4) Árbol botbi de India o de Sri Lanka.

5) Imagen del Buda hecha con piedra de montaña.

6) Reliquia de arahant.

7) Tierra de los sitios donde el Buda nació, se iluminó, enseñó el Dhammachaka y entró al nirvana.

8) Gran estanque Muchchalind.

En 2011, el joven abad de uno de los wat más importantes vinculados con Buth Savong me dijo que los elementos clave eran un conjunto del Tripitaka, una reliquia del Buda, un árbol bothi y, además del Tripitaka, un conjunto de los comentarios de Athakatha.

Los árboles bothi en los wat de Buth Savong se cultivan a partir de esquejes de árboles sagrados de Sri Lanka, o árboles que provienen en línea directa (esquejes de esquejes) de otros que se encuentran en Sri Lanka y que se considera provienen directamente de aquel bajo el cual el Buda alcanzó la iluminación.

Estos elementos adquieren mucha más prominencia de la que tendrían en otros wat camboyanos. Sea cual sea su importancia histórica más prolongada, la veneración de las reliquias y los árboles bothi ha sido mucho menos notoria en Camboya que en Sri Lanka y en otros países theravada en los años recientes (quizás desde la colonia francesa), y su adquisición en los wat afiliados con Buth Savong recibe mayor prioridad que la construcción de otras estructuras tradicionales en los wat. El Ministerio de Religión considera asram algunos de los sitios más importantes relacionados con Buth Savong, los cuales no cuentan con el edificio central grande, o vihara, que común- 
mente se emplea para rituales monásticos. ${ }^{13}$ En cualquier caso, la presencia o la ausencia de una vihara en sitios que se asocian con Buth Savong parece ser menos importante que la presencia de una stupa para reliquias, que se considera fundamental.

De hecho, los wat y los asram tienen otras características en común: una limpieza impecable, atención a los dormitorios de los monjes (construidos de tal manera que se puede monitorear fácilmente el comportamiento de los más jóvenes), comúnmente una casa pequeña designada para alojar a Buth Savong cuando pernocte ahí (aunque ello puede ser infrecuente o quizás incluso nunca suceda), y, con frecuencia, una estatua de él.

Lo que observo en todos estos atributos, ante la agitación social y religiosa que ha habido en Camboya en las últimas tres décadas, es un intento por crear un espacio sagrado en una forma diferente y más pura; además, veo la proliferación de wat y de asram afiliados a Buth Savong por todo el país, como un intento consciente de proyectar el espacio sagrado de la nación de una manera nueva.

Aunque la presencia de la Tripitaka puede considerarse ligeramente más importante, como lo indica su lugar en nuestra lista, las reliquias se han convertido poco a poco en uno de los elementos clave de los sitios relacionados con Buth Savong. Algunos de los sitios más importantes que alojan reliquias del Buda y están relacionados con Buth Savong ${ }^{14}$ son:

1) Asram Nikrothavan (Kol Totoeung, provincia de Kandal). 2) Wat Velovan (Prey Roeusey, distrito de Ang Snuel, provincia de Kandal).

${ }^{13} \mathrm{Al}$ parecer, Buth Savong decidió mantener dichos sitios como asram en lugar de convertirlos en wat, aunque esto no está totalmente claro. Nótese que el término vihara se emplea de manera diferente en el sureste de Asia que en Sri Lanka, donde se refiere al complejo del templo en su conjunto.

${ }^{14} \mathrm{El}$ abad del wat Velovan proporcionó esta lista en 2011. Es importante considerar que diversos wat de la lista pueden tener vínculos de muy diferente naturaleza con Buth Savong. Algunos de ellos pueden llamarse claramente wat de Buth Savong, en toda la extensión de la palabra. En el caso del wat Damnak, en Siem Reap, por ejemplo, la propia comunidad monástica del wat decidió construir una stupa para alojar una reliquia, que no sigue específicamente el patrón de los wat de Buth Savong en otros aspectos, aunque un predicador laico del dhamma, Keov Vimut, quien se considera discípulo de Savong, desempeñó una función en la adquisición de la reliquia, proceso que contó con el respaldo de Buth Savong. 
3) Asram Thudang (Chamka Mon, afueras de Phnom Penh).

4) Asram Preah Kanthakoti (anterior casa de Buth Savong, Battambang).

5) Wat Ratanak Pkay Preuk, Srok Ratanak Mondal, Battambang.

6) Wat S'ang Phnom Neang Len, Battambang.

7) Wat Konkea Kiri Baribatr, Battambang.

8) Wat Visotimakk.

9) Wat Damnak, Siem Reap.

10) Wat Preah Butthakhosachar, distrito de Oureng Ew, Kampong Cham.

11) Wat Tuol, Prey Veng.

12) Wat Chambak Mean Chey, Kandal.

13) Wat Prey Ko, Kandal.

14) Wat Prey Svay, Kampong Speu.

15) Wat Kirikoro.

16) Wat Prek Roeusey, distrito de S'ang, Kandal.

17) Wat Khpab, distrito de S'ang, Kandal.

La primera reliquia se instaló en el asram de Kol Toteoung, en 2001. Resulta sorprendente que, conforme se ha extendido la influencia de Buth Savong y cada vez más wat se afilian a él, también ha logrado introducir más y más reliquias a Camboya, de manera que hoy existen más de 200 de ellas en wat y asram vinculados a él, de acuerdo con un monje de alto rango del wat Velovan. El sitio web de Savong no hace referencia a este dato, pero la escala del movimiento de las reliquias adquiere dimensiones casi históricas. La coordinación de personas y la movilización de importantes recursos y esfuerzos personales para la recaudación de fondos destinados a la construcción de stupas y la instalación ceremonial de reliquias son significativas en sí mismas, pues constituyen inevitablemente una forma de integración social en torno de una meta religiosa, más allá del significado social más rutinario que la stupa y las reliquias adquieren una vez que se encuentran instaladas. Según la aplicación de Rappaport de la teoría del discurso a la antropología de la religión, ${ }^{15}$ la instalación de reliquias tiene una cualidad "performativa".

${ }^{15}$ Roy Rappaport, Ritual and Religion in the Making of Humanity, Cambridge, Cambridge University Press, 1999. 
Resulta evidente que el traslado de reliquias no puede ocurrir sin que haya fuentes fuera de Camboya que ofrezcan enviarlas, y parte del drama radica en que, independientemente de Buth Savong y su movimiento, en los últimos años se ha iniciado una circulación internacional más general de reliquias. Si bien esto puede relacionarse con la práctica reciente de realizar tours con reliquias tibetanas, por ejemplo, la mayoría de las que se envían a otros países provienen de Sri Lanka. (Algunas otras han llegado a Camboya desde India y Birmania, pero en menor medida.) No sólo se están destinando reliquias a Camboya, sino también a Tailandia y a templos étnicos vietnamitas y khmer en el delta del río Mekong, en Vietnam. Además, en 2010 se envió una reliquia al templo budista camboyano de Minnesota, Estados Unidos (aunque no está afiliado a Buth Savong). Sin embargo, esto escapa del ámbito de mi estudio. ${ }^{16}$

Consideraré tres casos específicos de stupas para reliquias de reciente construcción para ilustrar las dimensiones del fenómeno.

\section{Reliquia de Kol Totoeung}

La primera reliquia en arribar a Camboya en asociación con Buth Savong es la que se encuentra en el asram Nikrothavan, en Kol Totoeung, provincia de Kandal, a unos 45 minutos de Phnom Penh. Aunque lo más común es que las reliquias provengan de Sri Lanka, ésta es originaria de India. De acuerdo con un monje de alto rango que había estudiado en Sri Lanka, la adquirió un monje camboyano que vivía en Estados Unidos, San Savuth, quien la tenía en su wat en ese país pero a quien nunca le pareció haber encontrado un lugar permanente apropiado para ella. En 2001 se comunicó con una importante figura del budismo camboyano, la laica Nhek Buntha, quien había sido una alta funcionaria del Ministerio de Finanzas afiliada al PPC, que dejó la política y durante muchos años fue presidenta de la

${ }^{16}$ Hay una cierta correspondencia entre el florecimiento de las reliquias y el que Buth Savong haya comenzado a llevar grupos de peregrinos camboyanos a sitios sagrados en India y Sri Lanka. Parece lógico suponer que existe una conexión entre ambos hechos, pero no cuento con documentación clara al respecto. 
Asociación Budista Camboyana. Se le conoció en particular en su calidad de moderadora del Foro para el Entendimiento del Budismo, que transmitía pláticas y discusiones sobre el dhamma por televisión cada semana. Ella era cercana a Buth Savong y pensó que la reliquia sería perfecta para el complejo que él estaba construyendo en Kol Totoeung. La edificación de la stupa que alojaría la reliquia tuvo un costo de 400000 dólares y, en 2003, Nhek Buntha importó la reliquia desde Estados Unidos. Se realizó una procesión desde la casa de ella, en Phnom Penh, hasta el sitio del asram y una celebración religiosa que duró tres días con sus noches. La stupa para reliquias es interesante en tanto que su nivel inferior está abierto y el público puede entrar. Además, una gran imagen del Buda se ubica en la base de la stupa, que resulta impresionante porque las vetas del mármol le otorgan un aspecto de leproso. La reliquia se encuentra en la cúspide de la stupa, en una cámara a la que sólo es posible acceder a través de una escalera. La adquisición de la reliquia se llevó a cabo apenas un año antes de la conclusión de la enorme stupa nacional en Oudong, y la reliquia de Kol Totoeung se instaló un año después de que el rey trasladara las reliquias de la estación de ferrocarril a Oudong.

\section{Reliquia del wat Velovong}

Para cuando se depositó la reliquia en Kol Totoeung, ya había planes para construir una stupa llamativa en el wat Velovong, en el distrito Ang Snuol, provincia de Kandal, la cual se encuentra más o menos a la misma distancia de Phnom Penh, pero en otra dirección. La construcción de la stupa comenzó en 2004. En este caso, las reliquias provenían de Sri Lanka y tanto Buth Savong como el abad del wat y otros dirigentes religiosos de Sri Lanka organizaron su adquisición. Finalmente, una delegación de 40 personas, todas discípulas de Buth Savong, fueron a Sri Lanka a obtener las reliquias. La delegación incluía a diez monjes, entre ellos a Tim Saet, el segundo monje en jerarquía en Kol Totoeung; el resto eran personas adineradas y funcionarios del gobierno. No había representantes del Ministerio de Religión, aunque sí de su oficina provincial, en la ceremonia 
de inauguración de la stupa, en 2008. Un letrero que se encuentra sobre una de las puertas de entrada a la stupa indica, muy explícitamente, que costó 251205 dólares. Las tres reliquias son de dos tamaños; una de ellas es más grande que la mayoría de las que hay en Camboya.

Todo esto ocurrió a pesar del hecho de que el kannaq sang (el consejo regulador del monacato) comenzó a oponerse a que se trasladaran las reliquias a Camboya y emitió una proclamación monástica (sangha brakah), en 2004, que prohibía que se llevaran las reliquias a Camboya si no había prueba de que fueran auténticas. ${ }^{17}$ En una visita al wat Velovan, en 2006, me impresionó la escala de la construcción de la stupa, cuya intención era claramente la de formar parte de la misma constelación de santuarios budistas que la stupa de enormes proporciones que se encuentra en Oudong, patrocinada por el Estado. En mi visita, advertí que la palabra para nombrar a la stupa había cambiado de sakyamunchedi (que indica específicamente que alberga una reliquia del Buda) a baromachedi (el cual meramente sugiere que se trata de una stupa de gran poder espiritual) y especulaba si esto significaba que la reliquia ya no se ubicaría en ese lugar. Tal vez Buth Savong y su movimiento hayan guardado un perfil más bajo con ésta y otras stupas para reliquias de lo que habría sido de no ser por las sangha brakah. No obstante, la instalación de las reliquias en el baromachedi del wat Velovan prosiguió tal como se había planeado y siguieron colocándose nuevas reliquias a lo largo y ancho del país.

\section{Wat Sokharam Tramuong, distrito de Memot, Kampong Cham}

Las dos stupas para reliquias a las que acabo de hacer alusión son quizás las más famosas relacionadas con Buth Savong. Sería difícil hacer generalizaciones acerca del resto de las más de 200 reliquias que se encuentran en wat vinculados con él o de las

${ }^{17}$ Yo mismo hice referencia a otras reliquias en una entrevista sobre otro tema con un funcionario del ministerio que ahora es ministro de Religión, y me sorprendí por la vehemencia con la que insistía en que sólo hay una reliquia del Buda en Camboya. 
stupas que las alojan, pero sospecho que la mayoría siguen el patrón del wat Velovan en tanto que provienen de Sri Lanka, y ello implica una interacción con los cuerpos institucionales de ese país que tienen que ver con la distribución más amplia de reliquias en todo el mundo. El último caso que describo es un tanto más excéntrico. Lo describo porque es más colorido, muestra una vinculación con una oleada previa de distribución de reliquias en la década de 1950 y demuestra la serie de circunstancias bajo las cuales éstas se llevaron a Camboya. ${ }^{18}$

La reliquia en cuestión llegó de la provincia Vungtao, de Vietnam. De acuerdo con el abad del wat Sakharam Tramuong, había estado en poder de una mujer vietnamita de nombre Phrin Thi Co, quien guardaba los diez preceptos y que a pesar de ser vietnamita había residido durante muchos años en un wat Khmer Krom (de la etnia khmer). En 2008, cuando me entrevisté con el abad, él calculaba que ella ya debía tener aproximadamente 80 años, si aún estaba viva, y mencionó que nunca se casó ni tuvo hijos. La mujer tenía un sobrino que trabajaba en la administración colonial francesa.

En 1954, cerca de la época de la independencia, los franceses le permitieron a un monje khmer viajar a India junto con la monja vietnamita. Ella regresó con cuatro objetos: 1) una reliquia del Buda mismo; 2) una sombrilla color azafrán; 3) la reliquia de un arabant (alguien que ha alcanzado la iluminación), y 4) tierra de los lugares sagrados que había visitado y donde había orado: el lugar donde nació el Buda, el sitio donde alcanzó la iluminación, su Thammachaka, y el sitio desde donde entró al nirvana. De 1954 a 2002, dichos objetos permanecieron con ella en el wat Vung Tau. Durante esa época, Sam Tri, un hombre khmer que trabajaba en una plantación de hule, enfermó y acudió a la ciudad de Ho Chi Minh para recibir tratamiento. Un primo suyo que vivía allí lo llevó al lugar en el que se encontraban las reliquias, donde la monja confirmó su existencia. Muchos wat en la parte de Vietnam habitada por la etnia khmer habían pedido la reliquia, pero según el abad que relata la historia, siempre que se hacía una solicitud de este tipo sur-

${ }^{18}$ También es posible que este caso en particular haya tenido algo que ver con la proclamación monástica contra el traslado irregular de reliquias al país. 
gían pequeñas dificultades (como, por ejemplo, con la luz eléctrica que rodeaba el santuario donde se alojaba la reliquia) y la monja interpretaba eso como un signo de que no debía acceder. Sin embargo, cuando Sam Tri hizo esa misma petición, las luces no se apagaron; todas las autoridades del wat estuvieron de acuerdo con la decisión de la monja y permitieron que las reliquias se llevaran a Camboya. Los monjes de Memot llevaron a cabo una ceremonia para pedir que se abriera el camino a fin de que las reliquias llegaran allí sin problemas. La reliquia del Buda (junto con la reliquia del arahant, las cuatro muestras de tierra y la sombrilla) se trasladó en 2002 y se realizó una ceremonia de inauguración en 2003. Como temían la posible confiscación de las reliquias al cruzar la frontera, las escondieron en un sombrero; los oficiales fronterizos no notaron los recipientes donde estaban las reliquias. Se tomaron fotografías de la ceremonia de dedicación para mostrárselas a la monja en Vietnam, pero cuando se las llevaron, de acuerdo con el abad de Memot, ella dijo que no necesitaba verlas, pues ya había contemplado la ceremonia en una visión. Sam Tri invitó a Buth Savong a participar; Buth Savong subió por una escalera para colocar la reliquia del Buda en una cámara alta de la stupa. Todo esto sucedió poco después de que se hubieran depositado las reliquias en la enorme stupa de Oudong. El abad del wat Sakharam Tramuong también estuvo presente en dicha ocasión. Debemos destacar que la afiliación del wat Sakharam Tramuong con Buth Savong es relativamente marginal, y que los monjes de ahí no siguen estrictamente las medidas disciplinarias al estilo de Buth Savong. Al parecer, lo que ocurrió fue que éste prestó su nombre a un wat que contenía una reliquia, y no se llevó una reliquia a un wat afiliado a Buth Savong. Quizás esto no sea típico de las stupas para reliquias vinculadas a Buth Savong, pero forma parte del relato general.

En un trabajo anterior escribí cómo, en conjunción con las festividades del Buda Jayanti que se celebraban en Camboya en 1957 (2500 de la era budista), el rey Norodom Sihanouk llevó reliquias de Sri Lanka y las colocó en una stupa frente a la estación de trenes de Phnom Penh. Dado que este suceso fue contemporáneo a la independencia de Francia, empoderó a la monarquía y pareció establecer un nuevo orden cosmológi- 
co que de alguna extraña manera se combinó con una nueva visión de modernidad poscolonial. ${ }^{19}$ Después de la agitación del periodo del Pol Pot y del socialismo de la década de 1980, cuando Sihanouk regresó a Camboya a ocupar el trono, la decisión de construir una nueva stupa para las reliquias en un lugar más elevado y más sagrado también representó para muchos un hecho de significado cosmológico. (En esa misma época surgieron movimientos religiosos de las bases, concentrados en la construcción de monumentos religiosos, muchos de ellos en estilo "neoangkoreano", relacionados también con textos tradicionales milenarios que muchos creían que predecían los cataclismos que estaban ocurriendo en el país.) ${ }^{20} \mathrm{Si}$ las reliquias y las stupas que las contienen han jugado un papel menos significativo en el budismo camboyano que en otros países theravada, Ashley Thompson ${ }^{21}$ ha sugerido que podría deberse no tanto a una indiferencia hacia las reliquias, sino al hecho de que los vestigios de Angkor han servido en calidad de "reliquias" como un punto de referencia más importante.

La década de 1950 y el proceso de independencia de varios países budistas fueron testigos del ascenso del budismo internacional, que Sihanouk aprovechó cuando organizó los festejos del Buda Jayanti y trasladó reliquias importantes para instalarlas en el país. Esto era una celebración de la nueva "soberanía” de la "nación" y al mismo tiempo sirvió como una salvaguarda de la soberanía más literal de la monarquía camboyana.

El gesto de Sihanouk de llevar las reliquias y darles prominencia era consistente con los patrones históricos de un centro espiritual para una visión cosmológica de la monarquía camboyana, pero representaba una desviación de las tendencias del budismo camboyano durante el periodo colonial, en tanto que le dio una nueva prominencia a las stupas y las reliquias, acorde con la práctica budista en Sri Lanka y Birmania.

Después de años de socialismo, la decisión de construir un nuevo santuario para albergar la reliquia y la ceremonia

${ }^{19}$ John Marston, "El Buda Jayanti en Camboya”, Estudios de Asia y África, vol. XLIV (1), núm. 138, 2009, pp. 9-30.

${ }^{20}$ Véase John Marston, "Elaboración de narrativas de orden: construcción y caos moral”, Estudios de Asia y África, vol. xL (1), núm. 126, 2005, pp. 117-160.

${ }^{21}$ Comunicación personal, agosto de 2010. 
de 2002 para colocarla en la stupa de Oudong fueron gestos que se hicieron conscientemente para evocar su simbolismo y su poder en la nación. Buth Savong se basa en esta visión y la amplía, a la vez que, al brindar sitios alternos de devoción, su movimiento desafía la visión monolítica.

Es decir, el solo hecho de que Buth Savong maneje un vocabulario de reliquias indica que acepta el sistema cosmológicosimbólico del santuario de Oudong, y ciertamente se encuentra operando en esa esfera, con sus vínculos con el budismo internacional, en vez de con el simbolismo de Angkor Wat. Buth Savong extiende el vocabulario de la stupa de Oudong al grado de que la proliferación de sus santuarios representa también una extensión de su concepción del budismo: de pureza disciplinaria $\mathrm{y}$ de rigurosa atención a las escrituras budistas.

Su visión sustenta el orden social y, en general, ha sido bien recibida por el Estado y por los poderes monásticos. Pero el decreto del consejo monástico de que solamente debe haber una stupa con reliquias en Camboya sugiere que existe incomodidad en cuanto a su potencial como un poder espiritual alternativo. Las stupas relacionadas con Buth Savong también son instituciones donde se reciben donativos y se ganan méritos, que están levemente fuera de la tradición monástica dominante.

El concepto que tiene Wolters sobre la entidad política premoderna del sureste asiático como un "mandala" se basa en la idea de que la entidad política se concibió cosmológicamente. ${ }^{22}$ Las limitaciones de esta teoría radican en la amplia variedad de formas en que esto se puso en práctica a lo largo de la historia, una diversidad que desafía que se piense en ella en términos sencillos, como una única forma de organización social. En ocasiones implicó la asociación del poder de la monarquía, en el centro, con objetos protectores o elementos arquitectónicos monumentales y, frecuentemente, dentro del contexto budista, con la espiritualidad asociada con las reliquias y las stupas. Tambiah, al desarrollar el concepto en relación con una progresión histórica hacia la modernidad, empleó el término "entidad política galáctica pulsátil" para designar el "mandala" (donde a través del tiempo múltiples centros pueden resplandecer hacia

${ }^{22}$ Wolters, History, Culture, and Region in Southeast Asian Perspectives, op. cit. 
la prominencia), en contraste con la "entidad política radial”, la cual, con el paso del tiempo, imponía más rígidamente al centro sobre la periferia. ${ }^{23}$ Desde luego, Camboya es una entidad política moderna aunque, como pasa en cualquier cultura, su modernidad se ve complicada con las referencias al pasado.

Rappaport, ${ }^{24}$ con base en la teoría de los actos de habla, considera que el ritual es inherentemente performativo, en el sentido de que efectúa un cambio en las relaciones por el mero hecho de su representación. Me ha interesado el grado en el que estos proyectos de edificaciones religiosas también son "performativas" y cómo tal cualidad se pone en juego conscientemente en las conjunciones históricas de la Camboya contemporánea. El cambio histórico que está teniendo lugar en Camboya se presta a la representación dramática de la identidad espiritual, incluida la del proyecto estatal de construir una gran stupa en Oudong, pero también en los proyectos mediante los cuales los seguidores de Buth Savong están colocando reliquias y construyendo stupas.

Este artículo intenta reunir todas esas ideas para demostrar cómo la colocación de reliquias representa un acto de recrear performativamente el poder constituido en la forma del mandala, tal como lo describe Wolters. El interés de la campaña de Buth Savong para colocar reliquias en templos a lo largo y ancho de Camboya tiene que ver con la forma en la que conscientemente pone de manifiesto una nueva espiritualidad en el país, que abreva de una visión cosmológica (teñida con nociones de "mandala") para crear un espacio sagrado en un momento de cambio histórico.

\section{Traducción del inglés: María Capetillo Lozano}

Dirección institucional del autor:

Centro de Estudios de Asia y África

El Colegio de México

Camino al Ajusco 20

Pedregal de Sta. Teresa, 10740

${ }^{23}$ Tambiah, World Conqueror and World Renouncer, op. cit., p. 102.

${ }^{24}$ Rappaport, Ritual and Religion in the Making of Humanity, op. cit. 
México, D.F.

\jmars@colmex.mx

\section{Bibliografía}

Buth, Savong, Parents' Words of Wisdom, Phnom Penh, Dhamma4khmers.org, 2005.

Marston, John, "El Buda Jayanti en Camboya", Estudios de Asia y África, vol. XLIV (1), núm. 138, 2009, pp. 9-30.

MARSTON, John, "Elaboración de narrativas de orden: construcción y caos moral", Estudios de Asia y África, vol. XL (1), núm. 126, 2005, pp. 117-160.

Marston, John, “La reconstrucción de budismo 'antiguo' de Camboya”, Estudios de Asia y África, vol. XxxvII (2), núm. 118, 2002, pp. 271-303.

Poethig, Katherine, "Locating the Transnational in Cambodia's Dhammayātrā", en John Marston y Elizabeth Guthrie (eds.), History, Buddhism, and Nerw Religious Movements in Cambodia, Honolulú, University of Hawai’i Press, 2004, pp. 197-212.

RAPPAPORT, Roy, Ritual and Religion in the Making of Humanity, Cambridge, Cambridge University Press, 1999.

SHARF, Robert H., "On the Allure of Buddhist Relics", en David Germano y Kevin Trainor (eds.), Embodying the Dharma: Buddhist Relic Veneration in Asia, Albany, State University of New York Press, 2004, pp. 163-191.

Strong, John S., Relics of the Buddha, Princeton, Princeton University Press, 2004.

TambiaH, Stanley, World Conqueror and World Renouncer: A Study of Buddhism and Polity in Thailand against a Historical Background, Cambridge, Cambridge University Press, 1976.

TraINOR, Kevin, "Introduction beyond Superstition", en D. Germano y K. Trainor (eds.), Embodying the Dharma: Buddhist Relic Veneration in Asia, Albany, State University of New York Press, 2004.

Trainor, Kevin, Relics, Ritual, and Representation in Buddhism: Rematerializing the Sri Lankan Theravada Tradition, CambridgeNueva York, Cambridge University Press, 1997.

Wolters, O. W., History, Culture, and Region in Southeast Asian Perspectives, ed. rev., Ithaca, Cornell University Southeast Asia Program Publications, 1999. 